\title{
Factores de Riesgo Biopsicosociales para Enfermedad Renal Crónica en Estudiantes Universitarios
}

\author{
Biopsychosocial Risk Factors for Chronic Kidney Disease in University Students \\ Alexia Ostria-Vázquez, ${ }^{a}$, Rebeca M.E. Guzmán-Saldaña ${ }^{b}$, Gloria Solano-Solano $^{c}$, Rodrigo C. \\ León-Hernández ${ }^{d}$, Lilian E. Bosques-Brugada ${ }^{e}$ \& David Gutiérrez-Soria ${ }^{f}$
}

\begin{abstract}
:
The objective of the study was to know the relationship of biological, psychological and sociodemographic variables with the incidence of risk factors for Chronic Kidney Disease (CKD) in university students. The sampling was not probabilistic, Mexican university student's $N=200$ participated, with an age range of 18 to 25 years ( $\dot{X}=19.97$, D.E. $=1.73$ ). It was a cross-sectional nonexperimental study. Data were collected using blood and urine laboratory tests to determine the Glomerular Filtration Rate (GFR) using the CKD-EPI formula, a Food Consumption Frequency Questionnaire (CFCA), a Simple Self-Consumption of Water Consumption, the SISCO Academic Stress instrument, the Beck Anxiety Inventory (BAI) and sociodemographic data were recorded. The results were analyzed using descriptive statistics and Pearson's correlation. The study generated important information, to be considered in the implementation of intervention programs in health promotion and prevention of CKD from the early stages of life.
\end{abstract}

Keywords:

Chronic Kidney Disease, Risk Factors, Consumption of Sugary Drinks, University.

\section{Resumen:}

El objetivo del estudio fue conocer la relación de las variables biológicas, psicológicas y sociodemográficas con la incidencia de factores de riesgo para Enfermedad Renal Crónica (ERC) en estudiantes universitarios. El muestreo fue no probabilístico, participaron estudiantes universitarios mexicanos $\mathrm{N}=200$, con un rango de edad de 18 a 25 años ( $\dot{X}=19.97$, D.E.= 1.73). Se trató de un estudio no experimental transversal. Se recopilaron datos mediante pruebas de laboratorio de sangre y orina para determinar la Tasa de Filtrado Glomerular (TFG) mediante la fórmula CKD-EPI, un Cuestionario de Frecuencia de Consumo de Alimentos (CFCA), un Auto-registro de Consumo de Agua Simple, el instrumento SISCO de Estrés Académico, el Inventario de Ansiedad de Beck (BAI) y se registraron datos sociodemográficos. Los resultados se analizaron utilizando estadística descriptiva y correlación de Pearson. El estudio generó información de importancia, para ser considerada en la implementación de programas de intervención en promoción de la salud y prevención de ERC desde las primeras etapas de vida.

Palabras Clave:

Enfermedad Renal Crónica, Factores de Riesgo, Consumo de Bebidas Azucaradas, Universitarios.

\section{Introducción}

La Enfermedad Renal Crónica (ERC) constituye actualmente un problema de salud pública mundial debido al incremento en su incidencia y prevalencia (1). En México entre 1990 y 2010 se observó un incremento de más del $300 \%$ de la ERC lo cual afectó a ambos sexos y ocupó los primeros lugares de morbilidad y mortalidad (2).
Los sujetos con estadios tempranos de la enfermedad generalmente no son reconocidos ni tratados de manera oportuna, estos pacientes incrementan el riesgo de la pérdida de la función renal y el desarrollo de complicaciones (3), en este sentido, la ERC acorta la esperanza de vida, deteriora la calidad de vida de quienes la padecen y causa discapacidad laboral, que a su vez impacta a nivel económico (4).

\footnotetext{
${ }^{a}$ Universidad Autónoma del Estado de Hidalgo, Instituto de Ciencias de la Salud, Email: alexia.osv@gmail.com

${ }^{\mathrm{b}}$ Universidad Autónoma del Estado de Hidalgo, Instituto de Ciencias de la Salud, Email: remar64@yahoo.com.mx

${ }^{\mathrm{c}}$ Universidad Autónoma del Estado de Hidalgo, Instituto de Ciencias de la Salud, Email: gloriasol_20@yahoo.com.mx

${ }^{\mathrm{d}}$ Universidad Autónoma de Tamaulipas, Catedrático CONACyT, Email: rod_leonher@yahoo.com.mx

e Universidad Autónoma del Estado de Hidalgo, Instituto de Ciencias de la Salud, Email: lilian_bosques@uaeh.edu.mx

${ }^{\mathrm{f}}$ Universidad Autónoma del Estado de Hidalgo, Instituto de Ciencias de la Salud, Email: daguso72@ hotmail.com
} 
Las estrategias para mejorar el panorama de la ERC requieren de una intervención eficaz dirigida a la prevención primaria y hacia los estadios iniciales de la enfermedad, identificando los principales factores de riesgo de la progresión de la pérdida de la salud. Estos factores de riesgo están divididos en biológicos, psicológicos y sociales, los cuales pueden acelerar un daño renal. Generalmente se propone que existe una combinación de esos factores que interactúan sobre la función del riñón y provocan pérdida progresiva de la capacidad renal (5).

La falta de atención ante este problema de salud provoca desinformación y desinterés en la población, hasta el momento no ha sido posible establecer los mecanismos que aseguren la sustentabilidad de un programa de atención que combata el problema del daño renal, ni la educación sobre cuidados nefrológicos a edades tempranas dando como consecuencia detecciones tardías y el incremento de usuarios en tratamientos sustitutivos, que, por lo regular son de alto costo y que no todos tienen acceso a ellos (6).

El paso por instituciones educativas podría ser una herramienta para influir y apoyar a la población, como los programas de las "universidades saludables" que promueven estilos de vida saludables, fortaleciendo hábitos favorables para el cuidado de la salud (7), ya que las políticas públicas no han permitido enfrentar los nuevos desafíos epidemiológicos e incluso no existen modelos teóricos que expliquen la enfermedad en sus primeros estadios, por lo tanto, no hay programas eficaces y especializados en el diagnóstico oportuno en personas jóvenes antes que se propaguen las alteraciones renales (8).

Partiendo de estos antecedentes la presente investigación tuvo como objetivo general, conocer la relación de las variables biológicas, psicológicas y sociodemográficas con la incidencia de factores de riesgo para Enfermedad Renal Crónica en estudiantes universitarios.

Método

Tipo de estudio y diseño: Estudio explicativo con un diseño no experimental transversal.

Participantes: Se evaluaron a $\mathrm{N}=200$ alumnos universitarios hidalguenses, seleccionados mediante un muestreo no probabilístico por conveniencia. El rango de edad osciló entre los 18 y 25 años, la media de edad fue de 19.97 con una desviación estándar de 1.726 .

\section{Instrumentos:}

Cuestionario de Frecuencia de Consumo de Alimentos (CFCA), es un cuestionario que se articula en tres ejes fundamentales: una lista de alimentos, frecuencias de consumo en unidades de tiempo y una porción estándar establecida como punto de referencia para cada alimento. Diseñado apropiadamente puede evaluar la ingestión de alimentos de la dieta de una persona (9).

Registro de consumo de agua simple, es un registro realizado en forma de tabla a partir de imágenes con 10 vasos con agua por día de la semana, con un recuadro donde se pueden colocar el número de vasos u otra cantidad, se tiene que tachar la cantidad de vasos que consumen (10).

Inventario SISCO del estrés académico, el cuestionario tiene como objetivo central reconocer las características del estrés que suele acompañar a los estudiantes de educación media superior, superior y posgrado durante sus estudios. Creado y validado en México en 2007por Barraza-Macías (11). Cuenta con tres dimensiones: estresores, síntomas y estrategias de afrontamiento con un alfa de Cronbach de .90.

\section{Inventario de Ansiedad de Beck (BAI)}

Versión estandarizada en 2001 por Robles, Varela, Jurado \& Páez (12) para población mexicana $(\alpha=.83)$, es un auto informe de 21 ítems que evalúan síntomas de ansiedad en la semana previa a la aplicación, con cuatro opciones de respuesta. Los puntajes de severidad de síntomas van de 0 a 63.

\section{Fórmula CKD-EPI}

Su puntaje arroja el estadio de ERC a través de la Tasa de Filtrado Glomerular (TFG) medido con muestras de sangre y orina, detectando niveles de creatinina $y$ proteinuria (13).

\section{Formato sociodemográfico}

Cuestionario con aspectos generales de los participantes.

\section{Medidas antropométricas}

Índice de Masa Corporal (IMC), Índice de CinturaCadera (IC-C) y Presión Arterial (PA).

Análisis Estadístico: Se usó estadística descriptiva para conocer las medidas de tendencia central de los datos, correlación de Pearson para conocer las correlaciones entre la TFG y las variables biopsicosociales. 
Procedimiento: Se obtuvo la aprobación del protocolo de investigación por el comité de ética, posteriormente se acudió con las autoridades de los institutos universitarios solicitando su apoyo con los permisos pertinentes para la aplicación de las pruebas de laboratorio e instrumentos de medición. Se procedió a invitar a los universitarios, mostrándoles un consentimiento informado para establecer formalmente su cooperación. Los participantes que aceptaron fueron sometidos a la extracción de muestras sanguíneas y de orina para determinar la TFG; después se llevó a cabo la aplicación de batería de pruebas psicométricas y toma de medidas antropométricas. Los datos obtenidos fueron capturados en el programa estadístico SPSS versión 21 para llevar a cabo los análisis pertinentes de la investigación.

\section{Resultados}

Sexo: Mujeres 64\%, Hombres 36\%.

IMC: Bajo peso $6 \%$ Peso Normal $64 \%$, Sobrepeso u obesidad $30 \%$.

TFG: Estudiantes sin riesgo de ERC 29\% presentan TFG normal, estudiantes con riesgo de ERC 71\% presentan Hiperfiltración glomerular.

IC-C: Sin riesgo Cardiovascular $82 \%$, con riesgo cardiovascular $18 \%$.

Consumo de alimentos: El consumo semanal recomendado de frutas y verduras son 35 porciones, en promedio la muestra consume 23.78 porciones. El consumo semanal recomendado para alimentos altos en grasa y sodio son 7 porciones, en promedio la muestra consume 15.82 porciones.

Consumo de agua simple: Los vasos recomendados semanalmente son 56 vasos. En promedio los estudiantes beben 28.20 vasos. Los vasos recomendados semanalmente para bebidas azucaradas son 3.5 y en promedio la muestra bebe 15.38 vasos.

Estrés: Universitarios con niveles altos $65 \%$ y $35 \%$ con niveles bajos.

Ansiedad: Niveles bajos 55\% y con niveles altos $45 \%$.

Nivel socioeconómico: Nivel bajo $20 \%$, medio bajo $49 \%$, medio alto $19 \%$ y alto $12 \%$.

\section{Correlaciones}

Tabla 1.

Correlación de Pearson.

\begin{tabular}{|l|l|l|l|l|}
\hline Variables & Edad & IC-C & PAS & $\begin{array}{l}\text { Consumo } \\
\text { de agua } \\
\text { simple }\end{array}$ \\
\hline TFG & $-.282^{\star \star}$ & & & \\
\hline IMC & & $.477^{\star \star}$ & $.170^{\star}$ & $.173^{\star}$ \\
\hline PAD & & & $.590^{\star \star}$ & \\
\hline
\end{tabular}

Tabla 2.

Correlación de Pearson

\begin{tabular}{|l|l|l|l|l|}
\hline Variables & Ansiedad & Estrés & $\begin{array}{l}\text { Consumo } \\
\text { de Bebidas } \\
\text { azucaradas }\end{array}$ & $\begin{array}{l}\text { Consumo } \\
\text { de } \\
\text { Alimentos } \\
\text { con grasa } \\
\text { y sodio }\end{array}$ \\
\hline $\begin{array}{l}\text { Consumo } \\
\text { de agua } \\
\text { simple }\end{array}$ & $-.189^{* \star}$ & $-.175^{\star}$ & & \\
\hline $\begin{array}{l}\text { Ansiedad } \\
\text { IC-C }\end{array}$ & $-.200^{* *}$ & $-215^{\star *}$ & $.297^{* *}$ & $.239^{* *}$ \\
\hline $\begin{array}{l}\text { Consumo } \\
\text { de } \\
\text { Alimentos } \\
\text { con grasa y } \\
\text { sodio }\end{array}$ & & & $.669^{* *}$ & \\
\hline \begin{tabular}{l} 
Estrés \\
\hline
\end{tabular} & & & & \\
\hline
\end{tabular}

La única variable que se relacionó con la variable dependiente (TFG) fue Edad, se encontró una relación negativa baja y estadísticamente $(r=-.28, p=.01)$, donde a mayor edad menor será la TFG.

\section{Discusión}

La ERC por lo general se desarrolla de forma asintomática e incluso transcurre durante años, ya que la reserva renal es suficiente para mantener aparentemente sana a la persona afectada, aun cuando ésta se haya deteriorado hasta en $70 \%$, debido a lo anterior, cuando se diagnóstica la ERC ya hay un daño crónico importante de las nefronas dado durante un periodo de tiempo extenso (14). Lo anterior se explica desde el punto de vista que los síntomas sólo se detectan de manera temprana si se realizan las pruebas de laboratorio pertinentes, situación que rara vez ocurre a nivel nacional e internacional (15). Un claro ejemplo son los participantes de este estudio, ya que nunca habían realizado análisis pertinentes y no tenían 
conocimiento de su estado de salud renal e incluso al entregar los resultados de laboratorio algunos refirieron estar sorprendidos y preocupados ya que más del $50 \%$ tenía como diagnóstico hiperfiltración y no lo sabían.

Para conocer la prevalencia de la enfermedad se sugieren tamizajes para proporcionar datos concretos y diagnósticos oportunos (16), sin embargo, a pesar de la existencia de evidencias que resaltan la aparición de afectaciones renales en población joven y sin aparentes factores de riesgo, como en la ciudad africana de Kinshasa donde se reportó que de 263 estudiantes con una media de edad de 18.7 el $2 \%$ tienen ERC (17) pero a pesar de esto las instancias de salud pública no recomiendan hacer el tamizaje en la población general porque refieren no se ha demostrado que sea costoefectivo e incluso no existe un registro nacional de prevalencia e incidencia oficial $y$ aunque algunas investigaciones aportan información es complicado conocer cifras exactas.

La tarea para proporcionar un sistema de salud eficaz es fomentar el uso de los servicios gratuitos para educar a la población y que puedan practicarse análisis clínicos que detecten con mayor precisión factores de riesgo que promuevan el desarrollo de alguna enfermedad, ya que las condiciones de bienestar y de salud en general están estrechamente vinculadas con las condiciones de la vivienda, disponibilidad de alimentos y de ingreso, junto con la disponibilidad de adecuados servicios de salud y educativos que permitan un adecuado desarrollo en planteles educativos $y$ fomenten una adultez sana $(18,19)$.

\section{Conclusiones}

En este estudio el 1\% de los universitarios padece ERC y un $69 \%$ se encuentra con diagnóstico de hiperfiltración glomerular. Cifras alarmantes que sugieren estudiar más a fondo el fenómeno y ampliar datos para obtener un panorama más concreto acerca de la incidencia del padecimiento en poblaciones jóvenes.

En general, se encontró un porcentaje de universitarios considerable con un IMC elevado (30\%) en conjunto con una tendencia a riesgo cardiovascular medido a través del IC-C, bajo consumo de agua simple y consumo por encima de las recomendaciones nutricionales de bebidas azucaradas, en los hábitos alimentarios predomina la comida con altos contenidos de grasa y sodio y moderada frecuencia de consumo de frutas y verduras, la PA oscila en niveles óptimos.

Los resultados presentan evidencia de la importancia de la promoción de la salud en edades tempranas para evitar factores de riesgo para enfermedades crónicas. Se observaron niveles altos de estrés y ansiedad en un número considerable de los estudiantes. Resulta necesario analizar la planificación curricular y los tiempos de entrega de tareas, así como implementar consejerías y talleres para enseñar a los estudiantes a afrontar las situaciones estresantes y ansiosas.

Predomina el nivel socioeconómico medio-bajo en los universitarios, que es congruente a las cifras nacionales donde más del $50 \%$ de la población pertenece a este nivel, por lo tanto, es de suma importancia promover psicoeducación en el ámbito escolar y brindar herramientas para fomentar el cuidado de la salud, con campañas acordes a los niveles socioeconómicos que predominan en el país.

El perfil biopsicosocial de los universitarios arroja factores de riesgo latentes para el desarrollo de enfermedades crónicas. Estos datos son relevantes y demuestran la importancia de promover un cuidado integral de la salud desde etapas tempranas de desarrollo.

Los resultados obtenidos en esta investigación constituyen un antecedente para la Psicología de la Salud que permitirá desarrollar posteriormente intervenciones adecuadas que incidan en el autocuidado, revisiones y diagnósticos tempranos para eliminar complicaciones graves en la salud. Con estos avances se pretende que a futuro disminuyan gastos médicos por hospitalizaciones, invalidez laboral y mortalidad a causa de la ERC.

\section{Referencias}

[1] Couser WG, Remuzzi G, Mendis S, Tonelli M. The contribution of chronic kidney disease to the global burden of major noncommunicable diseases. Kidney Int. 2011;80(12):1258-70.

[2].Méndez-Durán A, Francisco Méndez-Bueno J, Tapia-Yáñez T, Montes AM, Aguilar-Sánchez L. Epidemiología de la insuficiencia renal crónica en México. Diálisis y Traspl. 2010;31(1):7-11.

[3] Lozano R, Gómez-Dantés H, Garrido-Latorre F, Jiménez-Corona A, Campuzano-Rincón JC, Franco-Marina F, et al. La carga de enfermedad, lesiones, factores de riesgo y desafíos para el sistema de salud en México. Salud Publica Mex. 2013;55(6):580-94.

[4] Flores JC. Enfermedad renal crónica: epidemiología y factores de riesgo. Rev Médica Clín Condes. 2010;21(4):502-7.

[5] Ávila-Saldivar MN. Enfermedad renal crónica: Prevención y detección temprana en el primer nivel de atención. Med Interna Mex. $2013 ; 29(2): 148-53$.

[6] Instituto Nacional de Salud Pública. Encuesta Nacional de Salud y Nutrición de Medio Camino 2016 Informe Final de Resultados.

[7] Becerra HS. Universidades saludables: una apuesta a una formación integral del estudiante. Rev Psicol. 2013;31(2):287-314

[8] National Kidney Foundation. K/DOQI clinical practice guidelines for chronic kidney disease: evaluation, classification, and stratification. Am J Kidney Dis. 2002; 39(2):1-26. 
[9] Curiel-Velázquez EN. Diferencias en la información obtenida en un cuestionario de frecuencia de consumo alimentario (CFCA) cuando se le da seguimiento a la dieta del escolar en relación con la obtenida de primera intención [Tesis]. México: Universidad Autónoma de Nuevo León;2012.

[10] Márquez-Estrada MV. Factores psicosociales asociados a la obesidad en escolares de Pachuca, Hidalgo [Tesis]. México: Universidad Autónoma del Estado de Hidalgo; 2013.

[11] Barraza-Macías A. El Inventario SISCO del Estrés Académico. Investig Educ Duranguense. 2007;1(7):89-93.

[12] Robles R, Varela R, Jurado S, Páez F. Versión Mexicana del Inventario de Ansiedad de Beck: Propiedades Psicométricas. Rev Mex Psicol [Internet]. 2001;18(2):211-8

[13] Bermúdez RM, Bover Sanjuán J, Oliver Samper A, Ballarín Castán JA, Gracia García S. Valoración de la nueva ecuación CKD-EPI para la estimación del filtrado glomerular. Nefrologia. 2010;30(2):185-94.

[14] Malaquias LC. Enfermedad Renal Cronica Y Su Atencion Medicnate Tratamiento Sustitutivo En México. UNAM. 2010;1(1):21-54.

[15] Orozco JAT, Quirós HSL. La enfermedad renal crónica en México HACIA UNA POLÍTICA NACIONAL PARA ENFRENTARLA. Mexico: Intersistemas; 2016.

[16] Fundación Mexicana del Riñón. Guías Latinoamericanas de práctica clínica sobre la prevención, diagnóstico y tratamiento de los estadios 1 5 de la Enfermedad Renal Crónica. 1st ed. CDMX: Programas Educativos; 2012

[17] Bukabau JB, Makulo J-RR, Pakasa NM, Cohen EP, Lepira FB, Kayembe PK, et al. Chronic kidney disease among high school students of Kinshasa. BMC Nephrol. 2012;13(1):13-24.

[18] Dupas P. Health Behavior in Developing Countries. SSRN. 2011; 3:1-39.

[19] Córdova-Villalobos JÁ, Barriguete-Meléndez JA, Lara-Esqueda A, Barquera S, Rosas-Peralta M, Hernández-Ávila M, et al. Las enfermedades crónicas no transmisibles en México: Sinopsis epidemiológica y prevención integral. Salud Publica de Mexico. 2008; 50(5):419-427 